\title{
La baja lira: de Masuccio Salernitano a Bernardo Tasso y Garcilaso
}

\author{
Isabel COLÓN CALDERÓN \\ Universidad Complutense de Madrid \\ isacolon@ucm.es
}

\section{RESUMEN}

Este artículo estudia el primer verso del poema de Garcilaso Ode ad florem Gnidi, "mi baja lira". El adjetivo "baja" está empleado de una forma diferente a otras obras, en que es utilizado de forma positiva. Antes de Garcilaso dos escritores presentaron como él la lira: Bernardo Tasso y Masuccio Salernitano. La relación de ambos autores con Nápoles apoya la conexión entre Garcilaso y la ciudad italiana.

Palabras clave: Garcilaso, Ode ad florem Gnidi, "baja lira", Bernardo Tasso, Masuccio Salernitano, Nápoles.

\begin{abstract}
This article studies the first line of Garcilaso's poem Ode ad florem Gnidi, "mi baja lira". The adjectif "baja" is diffrent from the descriptions normally applied to the word "lira", which way often associated with positive adjectives. Apart from Garcilaso, two earlier writers used "baja" to descrieb the lyre: Bernardo Tasso y Masuccio Salernitano. Both of these authors were from Nspoles, which is a further proof of the connection between Napoles and Garcilaso poetry.
\end{abstract}

Key words: Garcilaso, Ode ad florem Gnidi, "baja lira", Bernardo Tasso, Masuccio Salernitano, Naples. 


\section{Objetivos}

Mi intención en estas páginas es volver sobre las fuentes de la famosa expresión "baja lira" de la Ode ad florem Gnidi de Garcilaso, entre esas fuentes consideraré algunos textos que la crítica no ha tenido en cuenta, tanto en prosa como en verso.

\section{Posibles lecturas de Garcilaso}

Se pueden revisar los autores citados por el poeta toledano. Así, nos encontramos a Virgilio, el "Mantuano", en la Elegía II a Boscán ${ }^{l}$, y en la Égloga I², a Ovidio en "Pues este nombre perdí", dudosa, puesto que está en el epígrafe y puede no ser suya, al "vencedor latino", Julio César, en la Ègloga $I I^{4}$, a Petrarca, en la Epistola a Boscán ${ }^{5}$, a Tansillo 6 , Minturno y Bernardo Tasso en "Ilustre honor del nombre de Cardona", a Giulio Cesar Caracciolo en "Julio, después que me partí llorando $^{8}$ ", a Mario Galeota ${ }^{9}$, al que dedica el soneto XXXV, que sería el enamorado de la Canción $V^{10}$ y es mencionado en Ad Antonium Thylesium ode ${ }^{11}$, a Tilesio $^{12}$, varias veces a Boscán ${ }^{13}$, a Castiglione, en la carta a Jerónima Palova de Almogávar ${ }^{14}$, y se podría considerar a María de Cardona, elogiada como poetisa en la época ${ }^{15}$, aunque Chinchilla apunta la posibilidad de que en su origen Garcilaso se

\footnotetext{
${ }^{1}$ Garcilaso de la Vega (1995), p. 106, v. 3.

${ }^{2}$ Garcilaso de la Vega (1995), p. 129.

${ }^{3}$ Garcilaso de la Vega (1995), p. 8.

${ }^{4}$ Garcilaso de la Vega (1995), p. 207, v. 1474.

${ }^{5}$ Garcilaso de la Vega (1995), p. 119.
}

${ }^{6}$ Sobre la relación entre Tansillo y Garcilaso, M. Lefèvre (2006a), C. Hernando Sánchez (2003), pp. 94-95. Sobre la importancia de Tansillo en el petrarquismo español y napolitano, T. R. Toscano (2012).

${ }^{7}$ Garcilaso de la Vega (1995), p. 45. Sobre este poema, A. Gargano (2012) (a), pp. 175-177.

${ }^{8}$ Garcilaso de la Vega (1995), p. 36. Sobre Caracciolo, C. Hernando Sánchez (2003), p. 101 y G. Arbizzoni (2014).

${ }^{9}$ Sobre la identificación de Mario Galeota con Fabio Galeota, T. R. Toscano (2010), J. C. D’Amico (2011), p. 207 y J. Ponce Cárdenas (2012), nota 120. Sobre el tratado inédito de Galeota a propósito de la las fortificaciones, O. Brunetti (1999), pp. 229-230. Sobre sus vinculaciones con el valdesianismo, P. Lopez (1976).

${ }^{10}$ Garcilaso de la Vega (1995), p. 59, p. 86.

${ }^{11}$ Garcilaso de la Vega (1995), p. 250.

${ }^{12}$ Garcilaso de la Vega (1995), p. 245, p. 250. Sobre Tilesio, C. J. Hernando Sánchez (2003), p. 76 , p. 92 .

${ }^{13}$ Garcilaso de la Vega (1995), p. 52, p. 57, p. 106, p. 115.

${ }^{14}$ Garcilaso de la Vega (1995), p. 265.

${ }^{15}$ Garcilaso de la Vega (1995), p. 45. Sobre las relaciones entre María de Cardona e Isabella Villamarina, princesa de Salerno, y de esta con Garcilaso, E. Fosalba Vela (2009). Para la identificación entre una dama napolitana en la poesía de Garcilaso e Isabella Villamarina, B. Morros (2009). 
refiriese a Vittoria Colonna y Boscán introdujese una modificación ${ }^{16}$. También las composiciones en latín deben ser tenidas en cuenta, así Ginés de Sepúlveda aparece en Garsiae Lasi Ode ${ }^{17}$ y Antonio Silesio en Ad Antonium Thylesium ode ${ }^{18}$. En realidad son pocos los nombres a los que alude Garcilaso, directa o indirectamente.

Las citas son aun más raras; cabe recordar una de Petrarca, de la canción $\mathrm{Nel}$ dolce tempo, modificada ligeramente por Garcilaso en el soneto XXII ("Con ansia extrema"): "non esservi passato oltra la gona"19, y a Virgilio, en la carta a fray Jerónimo Seripando: "et crimine ab uno disc e omnis ${ }^{20 "}$.

Podemos detenernos, por otro lado, enlas semejanzas con los autores que la crítica se ha encargado de desvelar, y que es factible suponer que habría leído. En diferente grado de aproximación, y sin pretender por mi parte ninguna exhaustividad, se ha hablado, entre otros, de Horacio ${ }^{21}$, Lucrecio $^{22}$, Propercio ${ }^{23}$, Virgilio $^{24}$, Petronio ${ }^{25}$, Estacio ${ }^{26}$, Claudiano ${ }^{27}$, Marcial $^{28}$, Ausias March ${ }^{29}$, Dante ${ }^{30}$, Petrarca $^{31}$, Sannazaro ${ }^{32}$, Tansillo ${ }^{33}$, Bernardo Tasso ${ }^{34}, \mathrm{Bembo}^{35}$, así como de Garci Sánchez de Badajoz ${ }^{36}$ y Boscán ${ }^{37}$.

${ }^{16}$ R. H. Chinchilla (2008), p. 68.

${ }^{17}$ Garcilaso de la Vega (1995), pp. 252 y ss.

${ }^{18}$ E. Fosalba Vela (2012). Garcilaso de la Vega (1995), pp. 245-251.

${ }^{19}$ F. Petrarca (1984), I, p. 178, v. 34. Garcilaso de la Vega (1995), pp. 41-42, y pp. 400-401 sobre críticas a este uso de Garcilaso. Para la utilización de "versus cum auctoritate" en Petrarca y Garcilaso, A. Gargano (2008), p. 27.

${ }^{20}$ Garcilaso de la Vega (1995), p. 274.

${ }^{21}$ Garcilaso de la Vega (1995), p. 65, p. 73, p. 242. M. Menéndez Pelayo (1885), II, p. 14. P. N. Dunn (1981), pp. 130-143. A. Cruz (1981), p. 67.

${ }^{22}$ Garcilaso de la Vega (1995), p. 58.

${ }^{23}$ Garcilaso de la Vega (1995), p. 70.

${ }^{24}$ Garcilaso de la Vega (1995), p.73, p. 120.

${ }^{25}$ Garcilaso de la Vega (1995), p. 81.

${ }^{26}$ Garcilaso de la Vega (1995), p. 74. E. Fosalba Vela (2012), p. 141.

${ }^{27}$ Garcilaso de la Vega (1995), p. 147, p. 205, p. 229. A. Gargano (2012) (b).

${ }^{28}$ Garcilaso de la Vega (1995), p. 88.

${ }^{29}$ Garcilaso de la Vega (1995), p. 58, p. 70. Sobre Ausias March en Garcilaso, R. Lapesa (1985), pp. 62-70.

${ }^{30}$ A. Gargano (2008), p. 23.

${ }^{31}$ Garcilaso de la Vega (1995), p. 56, p. 62, p. 65, p. 72, p. 79, p. 81, p. 173. A. Gargano (2008), p. 27.

32 Garcilaso de la Vega (1995), p. 27, p. 63, p. 65.

${ }^{33}$ Garcilaso de la Vega (1995), p. 27, p. 45. Sobre Tansillo, M. Scotti (1973).

${ }^{34}$ Garcilaso de la Vega (1995), p. 27, p. 43, p. 57, p. 99, p. 100, p. 102, p. 104, p. 105.

${ }^{35}$ Garcilaso de la Vega (1995), p. 78, p. 88, p. 203.

${ }^{36}$ Garcilaso de la Vega (1995), p. 55, p. 85. Sobre la presencia de la poesía de cancionero en Garcilaso, R. Lapesa (1985), pp. 53-62.

${ }^{37}$ Garcilaso de la Vega (1995), p. 22, p. 71. 
La mayoría de las obras en las que se habría inspirado Garcilaso son líricas, aunque se han recordado textos en prosa o narrativos que habrían dejado huella en las composiciones garcilasistas, como De bello gallico de Julio César ${ }^{38}$, las Historias de Tácito $^{39}$, la Eneida ${ }^{40}$ y las Bucólicas ${ }^{41}$ de Virgilio, la Arcadia de Sannazaro $^{42}$, las Metamorfosis de Ovidio ${ }^{43}$, o el Orlando furioso de Ariosto ${ }^{44}$, entre otros. Estaríamos, por tanto, ante un tipo de imitación cruzada ${ }^{45}$.

Es posible que Garcilaso leyera textos de autores que no cita, y que la crítica no ha anotado. No están todas las obras a las que se acercó en el inventario de sus libros, o en los de su familia ${ }^{46}$; pudo tener en sus manos textos ajenos, sobre todo si tenemos en cuenta su estancia en Italia, y ya antes su relación con humanistas italianos en Toledo ${ }^{47}$ y pudo consultar otras bibliotecas, como la del virrey de Nápoles Pedro de Toledo ${ }^{48}$.

\section{Epítetos negativos para la lira en la poesía}

Para la Canción $V$ de Garcilaso, la Ode ad florem Gnidi, se han propuesto diversas fechas. Rafael Lapesa afirma que corresponde a la etapa italiana, desde septiembre de 1532; para Bienvenido Morros fue compuesta en Nápoles entre 1533 y 1536 , mientras que Dunn reduce el marco temporal a los años $1535-1536^{49}$.

En la primera estrofa leemos:

Si de mi baja lira tanto pudiese el son, que en un momento aplacase la ira del animoso viento

${ }^{38}$ Garcilaso de la Vega (1995), p. 227.

${ }^{39}$ Garcilaso de la Vega (1995), p. 213.

${ }^{40}$ Garcilaso de la Vega (1995), p. 120, p. 274.

${ }^{41}$ Garcilaso de la Vega (1995), p. 240, p. 241.

${ }^{42}$ Garcilaso de la Vega (1995), p. 121, p. 170, p. 171. Sobre la influencia de la Arcadia en Garcilaso, M. Lefèvre (2006b) y A. Gargano (2011).

${ }^{43}$ Garcilaso de la Vega (1995), p, 122, p. 228.

${ }^{44}$ Garcilaso de la Vega (1995), p. 123.

45 A. Cruz (1988), p. 91.

${ }^{46}$ Se pueden ver en K. Sliwa (2006), pp. 156-159, pp. 189-190, p. 310.

${ }^{47}$ E. Fosalba Vela (2012), pp. 133 y ss.

${ }^{48}$ C. J. Hernando Sánchez (1980). C. J. Hernando Sánchez (1994), pp. 477-483.

${ }^{49}$ R. Lapesa (1985), p. 188. Garcilaso de la Vega (1995), p. 84; P. N. Dunn (1981), p. 142. Sobre la estancia de Garcilaso en Nápoles, y en general en Italia, E. Mele (1923), pp. 108148; C. J. Hernando Sánchez (1994), pp. 486-488; C. J. Hernando Sánchez (2004); M. Lefèvre (2006c), pp. 55-59 y E. Fosalba Vela (2009). Sobre Nápoles, C. J. Hernando Sánchez (1994) y (2004) y G. Galasso (2000). 
la furia del mar y el movimiento ${ }^{50}$.

Hay que recordar que Garcilaso usó el adjetivo "bajo" en alguna ocasión más, pero referido a un intrumento de otro tipo, así en la Égloga III el poeta se dirige a una dama, "ilustre y hermosísima María", tal vez la mujer del virrey de Nápoles, a la que pide en los versos 42 y 43 :

Aplica, pues un rato los sentidos

al bajo son de mi zampoña ruda ${ }^{51}$.

Se han buscado posibles inspiraciones para la expresión "baja lira". Así, se ha recordado a Horacio, I, 6 aunque según Dunn Garcilaso no sigue la humildad, real o fingida, del poeta latino, y se dirige a Agripa, no a una dama ${ }^{52}$, y a Propercio II, $1^{53}$.

Se ha puesto de relieve que la lira normalmente gozaba de una consideración positiva ${ }^{54}$, y se han propuesto interpretaciones para el uso de un adjetivo supuestamente ajeno a la tradición, así Herrera lo explica como: "Humildad y extenuación de su ingenio" ${ }^{55}$; sin embargo, el instrumento musical sí recibió valoraciones negativas en verso, en Pietro Jacopo Di Jennaro y en Bernardo Tasso, $\mathrm{y}$, como veremos, en prosa.

El napolitano Pietro Jacopo Di Jennaro, muerto en $1508^{56}$, se sirvió en su Canzoniere de términos no elogiosos para referirse a su escritura y sus capacidades $^{57}$. Por ejemplo, alude a "acerbo scriver mio"58, "bascio intellecto",59, "rime aspre et humile ${ }^{60 ",}$, "son rauco ${ }^{61 "}$ y "mio stil debele ${ }^{62}$ ", algunas de cuyas

${ }^{50}$ Garcilaso de la Vega (1995), p. 84.

${ }^{51}$ Garcilaso de la Vega (1995), p. 226. Sobre la identificación de María con la mujer del virrey de Nápoles, Garcilaso de la Vega (1995), p. 223.

${ }^{52}$ P. N. Dunn (1981), p. 131, p. 138. Sobre Horacio I, 8, F. Lázaro Carreter (1986), p. 121. Horacio (1990), p. 100 y p. 106.

${ }^{53}$ F. Lázaro Carreter (1986), p. 115. Se trata de "non ego Titanas canerem". Propercio (1984), p. 138, v. 19.

${ }^{54}$ F. Lázaro Carreter (1986), p. 116. Garcilaso de la Vega (1995), p. 84, p. 432.

${ }^{55}$ Garcilaso de la Vega (1995), p. 84. Garcilaso de la Vega (1972), p. 410.

${ }^{56}$ M. Santagata (1979), p. 10. Sobre Di Jennaro, M. Santagata (1979), pp. 280-281, p. 284, pp. 286-295, pp. 357-360, p. 390; Di Dio (2009).

${ }^{57}$ Sobre la valoración negativa de las propias obras, E. R. Curtius (1976), I, pp. 127-131 y I. Colón Calderón (2007), p. 677, p. 679.

${ }^{58}$ P. Jacobo Di Jennaro (1883), p. 69 ("Non Calyope, Euterpe, Urania et Clyo”, v. 5).

${ }^{59}$ P. Jacopo Di Jennaro (1883), p. 106 ("Le labia che cantar sovente in versi”, v.10).

${ }^{60}$ P. Jacopo Di Jennaro (1883), p. 302 ("Alto sugiecto al mio infimo stile", v. 8).

${ }^{61}$ P. Jacopo Di Jennaro (1883), p. 358 ("Conosco ben che sbegoctito et smorto", v. 51).

${ }^{62}$ P. Jacopo Di Jennaro (1883), p. 381 ("Poi che letoi e beate orecchie intendere", v. 6).

Sobre este poema M. Santagata (1979), p. 381. 
afirmaciones podrían incluirse en la tradición que se ha llamado petrosa ${ }^{63}$. Por otro lado, siguió un sistema similar con la lira, ya que le atribuye características poco halagüeñas: "Tentar la roza et discordante lira ${ }^{64 ",}$ aunque no empleó con ella "bascia", que sí había aplicado al intelecto.

Adquiere especial importancia el caso de Bernardo Tasso, amigo de Garcilaso ${ }^{65}$, en cuya lírica vemos algunas semejanzas más estrechas con el toledano, en fechas próximas a la Canción $V$.

El poeta italiano expresó en algunas ocasiones una aparente falta de apreciación hacia su propia lírica. Así, en el libro primero de Gli Amori, publicado en $1531^{66}$, en "Sacro arbuscel, che 'l caro amato nome", alude a "incolto e basso stile"67, así como en "Se'l duro suon di que'sospiri ardenti ${ }^{68 \text { ", }}$ que Zampese ha puesto en relación con poemas de Jennaro, Cariteo y Trissino, a la vez que subraya su oposición a la inspiración proveniente de las Musas de alguna composición de Bembo ${ }^{69}$. En el soneto dirigido a Bembo, "Bembo, che d'ir al ciel mostri il camino", en el verso 9 se considera de "tardo ingegno ${ }^{70 "}$ ".

Por otro lado, en el libro tercero de sus Gli amori, publicado en 1537, en la estancia LXVIII, Tasso se refiere a su "basso ingegno", y utiliza "lira" con el adjetivo negativo "roca", de nuevo en la tradición pertrosa:

Temo, Donna gentil, ch'abbiate a sdegno

che canti più di voi si roca lira, poscia ch'alti concetti al basso ingegno il vostro gran valor più non inspira ${ }^{71}$.

Además, en la estancia LIV de Per la Signora Giulia Gonzaga del mismo libro III, le pide a la dama:

tanto valor a la mia lingua inspira,

\footnotetext{
${ }^{63}$ A. Gargano (2008), pp. 23-26.

${ }^{64}$ P. Jacopo Di Jennaro (1883), p. 244 ("Non par ch'ardisca la mia mano algente”, v. 2).

${ }^{65}$ Garcilaso de la Vega (1995), p. 45.

${ }^{66}$ B. Tasso (1995), I, p. 19.

${ }^{67}$ B. Tasso (1995), I, p. 19. Sobre el poema, C. Zampese (2012), p. 31. Garcilaso de la Vega (1995), p. 432.

${ }^{68}$ B. Tasso (1995), I, p. 101. Sobre los cambios de posición que afectaron a "Se'l duro suon di que'sospiri ardenti" en las ediciones de 1531 y de 1534, B. Tasso (1995), I, p. 101, pp. 413-414, G. Ferroni (2011), p. 99 y C. Zampese (2012), p. 29. Sobre el poema, G. Ferroni (2011), p. 99, p. 101. C. Zampese (2012), pp. 28-33.

${ }^{69}$ C. Zampese (2012), p. 30 (Jennaro), p. 30 (Cariteo), pp. 29-30 (Trissino), pp. 29-33 (Bembo).

${ }^{70}$ B. Tasso (1959), i, p. 23. Sobre el poema, G. Cerboni Baiardi (1986), pp. 46-47.

${ }^{71}$ B. Tasso (1995), I, p. 387.
} 
che 'l tuo solo favor invoca e chiede, ch'al roco suon di questa bassa lira possa cantar a chi non gli ha veduti i rari effetti de le tue vertuti ${ }^{72}$.

Nos encontramos en esta estrofa con el verso "ch'al roco suon di questa bassa lira", muy parecido al de Garcilaso, así como una construcción condicional en "Se'l duro suon di que'sospiri ardenti", y siempre dentro de la línea de los términos petrosos que hemos visto en Di Jennaro.

Se aprecia, pues cierta similitud entre Tasso y Garcilaso, aunque es difícil señalar la precedencia cronológica de uno u otro, $y$, como veremos, hay que tener en cuenta la prosa novelística.

"Bassa lira" no está en Ravisio Textor, según ha indicado Lázaro Carreter ${ }^{73}$, pero en las recopilaciones de epítetos tomados de autores italianos se incluye precisamente "bassa" como uno de los adjetivos característicos de "lira". Así en el Giardino degli epiteti, traslati et aggiunti poetici italiani de 1665, aludiendo a la estancia LIV de Per la Signora Giulia Gonzaga de Tasso, al igual que "roca lira"74.

Es decir, Garcilaso pudo inspirarse en Tasso, que a su vez habría recordado a Di Jennaro.

\section{La baja lira de Masuccio y Garcilaso}

Pero, además, una "bassa lira" había aparecido, antes de Garcilaso y Tasso, en un texto narrativo, en concreto en Il Novellino de Masuccio Salernitano, donde se insiste en las supuestas dificultades del autor para componer historias que estuviesen a la altura de aquellos a los que iban dirigidas, con mención expresa de la lira.

Il Novellino apareció póstumo en 1476, y aunque la edición princeps se ha perdido, se publicó luego en varias ocasiones, algunas de las cuales pudo conocer Garcilaso $^{75}$.

Il Novellino tiene una estructura compleja ${ }^{76}$, especialmente en lo concerniente a las dedicatorias, aunque no sigue el sistema boccacciano.

Se encuentra dividido en 5 partes (I-X; XI-XX; XXI-XXX; XXXI-XL; XLI-L). Todo el libro, así como la novela XLIV ${ }^{77}$, está dirigido a una única mujer, Hipólita

${ }^{72}$ B. Tasso (1995), I, p. 383.

${ }^{73}$ F. Lázaro Carreter (1986), p. 116.

${ }^{74}$ G. B. Spada (1665), p. 425 , p. 426.

${ }^{75}$ Sobre ediciones de Masuccio, T. R. Toscano (1998), p. 153, D. Berruezo (2012), p. 140, D, Berruezo (2013), pp. 97-100, M. Fusilli (2013), p. 119. D. Berruezo (2015) señala su presencia en cuentos, novelas y teatro, pero no en poesía.

${ }^{76}$ Sobre la estructura, G. Petrocchi (1953), pp. 54-57, D. Pirovano (1996), pp. 8-15,T. R. Toscano (1998), p. 152, L. Terrusi (2011), pp. 149 y ss. y M. Fusilli (2013), p. 120. 
María de Aragón y Visconti, o Hipólita Sforza, duquesa de Calabria, mujer de Alfonso, duque de Calabria, que era el primogénito del rey Ferrante $\mathrm{I}^{78}$.

Hay primero una dedicatoria general, con una aclaración del autor ("Masuccio"), a continuación una breve explicación del novelista al comienzo de las partes (salvo de la primera), luego se incluyen los argumentos de cada historia, una dedicatoria particular ("Esordio"), dirigida a una figura diferente, todas de la corte de Aragón en Nápoles ${ }^{79}$. Y por fin se desarrolla la narración, completada por unas palabras de Salernitano ("Masuccio), en las que puede hablar otra vez con Hipólita (por ejemplo, en la novela I, dedicada a Fernando de Aragón y en la de la XLIV, a ella dirigid ${ }^{80}$ ). El libro concluye con un "Parlamento de lo autore al libro suo".

Si se hace un repaso de las formas despreciativas con que Masuccio valora su producción, encontramos varias de ellas, dentro de la tradición petrosa, aunque hay que tener en cuenta la escasa "fiabilidad de su traicionado texto", en palabras de Toscano $^{81}$.

Masuccio en la dedicatoria general a Hipólita acumula las consideraciones desfavorables: piensa que su "bassa e rauca lira non si convenga de libro comporre ${ }^{82 "}$, califica a su ingenio de "grosso e rudissimo ${ }^{83 "}$ y habla de su "pigra y rozza mano ${ }^{84 ", ~ d e ~ l a ~ c u a l ~ h a ~ s a l i d o ~ u n ~ " l i b r e t t o " ~}{ }^{85}$, término que Terrusi pone en relación con el usado por Boccaccio en "novellete"86, de ahí que lo haya llamado $\mathrm{Il}$ Novellino; pero, a pesar de todo ello, le ruega a la duquesa que acoja sus novelas en la "tua sublime e gloriosa biblioteca ${ }^{87}$ ".

La supuesta insuficiencia de su pluma para escribir el "libretto" se revela asimismo en la dedicatoria a la novela XVIII, a Antonio de Sanseverino, primogénito del príncipe de Salerno, Roberto de Sanseverino. En este caso habla de su "insofficiente lira ${ }^{88 " .}$

${ }^{77}$ M. Salernitano (1975), pp. 3-6 y p. 346.

${ }^{78}$ L. Terrusi (2011), p. 149. Sobre los cambios en los apellidos de Hipólita, L. Terrusi (2011), p. 150. Sobre poemas dedicados a Hipólita, M. Santagata (1979), p. 15, p. 67, p. 249.

${ }^{79}$ M. I. Guijosa (2010), p. 171. Sobre la importancia de las dedicatorias, G. Petrocchi (1953), pp. 57-63.

${ }^{80}$ M. Salernitano (1975), p. 17, p. 353. Es mencionada también en M. Salernitano (1975), p. 181 (palabras preliminares de la III parte).

${ }^{81}$ T. R. Toscano (1998), p. 154.

${ }^{82}$ M. Salernitano (1975), p. 3

${ }^{83}$ M. Salernitano (1975), p. 3

${ }^{84}$ M. Salernitano (1975), p. 3

${ }^{85}$ M. Salernitano (1975), p. 3.

${ }^{86}$ L. Terrusi (2011), p. 151. G. Boccaccio (2013), p. 685 (IV, Introducción, 3).

${ }^{87}$ M. Salernitano (1975), p. 3. Sobre la presencia de Masuccio en los libros de los reyes aragoneses en Nápoles, J. Monfrin (1956), p. 204.

${ }^{88}$ M. Salernitano (1975), p. 158. 
En las palabras preliminares a la cuarta parte Masuccio comenta la "presente operetta ${ }^{89}$

En la dedicatoria a Francesco Galeota ${ }^{90}$, en la novela XLI, opone la "dulcissima lira" de Galeota a su "ruda mano ${ }^{91 "}$.

En la alocución posterior a la novela XLIV, de nuevo dirigida a Hipólita de Aragón, afirma que "sento raoca la mia lira, debile cognosco l'ingegno, e la ruzza mano insufficiente" 92 , y en la que cierra la novela XLV, dedicada a Enrico de Aragón, se considera de "bascio giudicio"93.

En el Parlamento final retoma el término "libretto", al que califica de "umile", y lo llama "povere Novellino" 94 .

Por otro lado, en la dedicatoria a la novela IV, a Antonello de Petruciis, evoca la lira de Orfeo, cuyas habilidades él no tiene: "se in me fosse la lira de Orfeo o la eloquenzia di Mercurio, non altramente che un vil canto" ${ }^{15}$. Y en la dedicatoria a la XLI, dirigida a Francesco Galeota, es Anfión el personaje aludido: "E se de la suave musica d'Anfione fuorno le dure pietre commose, nobilissimo mio Galioto, quele mareviglie $[\ldots]^{\prime \prime} 96$.

Es decir, la lira de Masuccio es "bassa", "rauca", "insofficiente" y "raoca", su ingenio resulta "grosso", "rudissimo" y "debile", su juicio "bascio", su mano "pigra", "rozza", "ruda" y "ruzza", y su librillo, "umile".

Tenemos, por lo tanto, coincidencias entre la Canción $V$ de Garcilaso y Masuccio en diferentes campos. Se encuentran epítetos negativos en los dos escritores asociados a la lira ("bassa", "rauca" y "raoca"/ "baja"97), y uno idéntico ("bassa"); alguno positivo, "suave musica" en Masuccio, "süave canto" en Garcilaso ${ }^{98}$; hay construcciones condicionales en Masuccio, tanto en la dedicatoria a la novela IV ("se in me fosse la lira de Orfeo"), como en la XLI ("E se de la suave musica d'Anfione fuorno le dure pietre commose"), y en Garcilaso ("Si de mi baja lira"); además, ambos parecen anhelar la habilidad musical de personajes mitológicos.

${ }^{89}$ M. Salernitano (1975), p. 251. Sobre el cambio que se produce en esta parte, D. Pirovano (1996), p. 13.

${ }^{90}$ E. Percopo (1895), pp. 17 y ss, M. Salernitano (1975), p. 415, M. Santagata (1979), pp. 180-181, pp. 388-389. Sobre la amistad de Galeota y Masuccio y los poemas del primero al segundo Altamura (1978).

${ }^{91}$ M. Salernitano (1975), p. 322.

${ }^{92}$ M. Salernitano (1975), p. 353.

${ }^{93}$ M. Salernitano (1975), p. 361.

${ }^{94}$ M. Salernitano (1975), p. 400, p. 404.

${ }^{95}$ M. Salernitano (1975), p. 41.

${ }^{96}$ M. Salernitano (1975), p. 322.

${ }^{97}$ Hay que recordar la "zampoña ruda" de la Égloga III.

${ }^{98}$ Garcilaso de la Vega (1995), p. 85 (v. 7). 


\section{Conclusiones}

La lira, por lo tanto, recibió epítetos negativos, en ocasiones dentro de la tradición petrosa. Pietro Jacopo Di Jennaro habló de "roza et discordante lira"; Masuccio Salernitano, en la dedicatoria general a Hipólita del Novellino, considera metafóricamente que posee una "bassa e rauca lira"; Bernardo Tasso en Per la Signora Giulia Gonzaga repite los vocablos: "roco suon di questa bassa lira". Garcilaso en "Si de mi baja lira/tanto pudiese el son" se integra en la línea napolitana de desvalorización de la lira, además de iniciar la consideración de la palabra "lira" como estrofa.

\section{Obras citadas}

ALTAMURA, Antonio: "Moduli masucciani in una novella inedita di Francesco Galeota", en Pietro Borrarko y Francesco D'Episcopo (eds.): Masuccio. Noveliere salernitano dell'eta' Aragonese, Galatina, Congedo Editore, 1978, I, pp. 135-147.

ARBIZZONI, Guido: "Le rime di Giulio Cesare Caracciolo in un nuovo manoscrito d'autore", en Giorgio Piras (ed.): Labor in Studiis. Scritti di filologia in onore di Piergiorgo Parroni, Roma, Salerno Editrici, 2014, pp. 227-296.

BERRUEZO SÁNCHEZ, Diana: "Il Novellino de Masuccio Salernitano en algunas comedias de Lope y Calderón", en Sònia Boadas, Félix Ernesto Chávez y Daniel García Vicens (eds.): La tinta en la clepsidra. Fuentes, historia y tradición en la literatura hispánica, Barcelona, PPU, 2012, pp. 139-149.

BERRUEZO SÁNCHEZ, Diana: "Sobreviviendo a la censura: Masuccio Salernitano en las letras castellanas", en Teresa Navarrete Navarrete y Miguel Soler Gallo (eds.): El eterno presente de la literatura. Estudios literarios de la Edad Media al siglo XIX, Roma, Aracne, 2013, pp. 97-106.

BERRUEZO SÁNCHEZ, Diana: Il Novellino de Masuccio y su influencia en la literatura española de la Edad de Oro, Vigo, Editorial Academia del Hispanismo, 2015.

BOCCACCIO, Giovanni: Decameron, ed. Amedeo Quondam, Maurizio Fiorilla y Giancarlo Alfano, Milano, BUR, 2013.

BRUNETTI, Oronzo: "La pratica dell'architettura militare nel viceregno di Napoli del XVI secolo", Boletín del Seminario de Estudios de Arte y Arqueología BSAA de la Universidad de Valladolid, LXV(1999), pp. 219-235.

CERBONI BAIARDI, Giorgio: La lirica di Bernardo Tasso, Urbino, Argalia, 1986.

CHINCHILLA, Rosa Helena: "La inspiración poética, la musa divina, el mecenazgo femenino en la obra de Garcilaso", en Pedro Ruiz Pérez (ed.): Canones críticos en la poesía de los Siglos de Oro, Vigo, Academia del Hispanismo, 2008, pp. 65-74. 
COLÓN CALDERÓN, Isabel: "Pío II y algunos tópicos proemiales en la España del XVI", en Luisa Secchi Tarugi (ed.): Pio II umanista europeo, Firenze, Franco Cesati, 2007, pp. 671-683.

CRUZ, Anne, Imitación y transformación: el petrarquismo en la poesía de Boscán y Garcilaso de la Vega, Amsterdam, John Benjamins, 1988.

CURTIUS, Ernst Robert: Literatura europea y Edad Media latina, México/ Madrid/Buenos Aires, Fondo de Cultura Económica, 1976.

D’AMICO, Juan Carlos: "Charles Quint, Pedro de Tolède et les émeutes napolitaines de 1547", en Pierre Civil, Antonio Gargano, Matteo Palumbo y Encarnación Sánchez García (eds.): Fra Italia e Spagna. Napoli crocevia di culture durante il vicereame, Napoli, Liguori, 2011, pp. 181-209.

DI DIO, Alessia: "Tipologie tematiche di sonetti amorosi. Il canzonieri di De Jennaro", Lettere italiane, LXI, 2(2009), pp. 177-219.

DI JENNARO, Pietro Jacopo: Il Canzoniere, ed. Giuseppe Barone, Napoli, Anotonio Morano, 1883.

DUNN, Peter N.: "La oda de Garcilaso A la flor de Gnido. Comentario a algunos temas e ideas del Renacimiento", en Elias L. Rivers (ed.): La poesía de Garcilaso, Barcelona, Ariel, reimpresión 1981, pp. 129-162.

FERRONI, Giovanni: "Como legere I tre libri degli Amori di Bernardo Tasso (1534-1537)", Quaderno di italianistica 2011, Pisa, ETS, [2011], pp. 99-144.

FOSALBA VELA, Eugenia: "Implicaciones teóricas del alegorismo autobiográfico en la égloga III de Garcilaso. Estancia en Nápoles", Studia Aurea, 3(2009), pp. 39-104.

FOSALBA VELA, Eugenia: "Sobre la relación de Garcilaso con Antonio Silesio y el círculo de los hermanos Seripando", Cuadernos de Filología italiana, 19(2012), pp. 131-144.

FUSILLI; Michele: "Racontando l'ore e igiorni. Bandello narratore tra Rime e Novelle, London, Royal Holloway College, 2013. Se puede leer en https://repository.royalholloway.ac.uk/file/b148f7a0-1088-7eb7-dde2e6a6274ce19c/7/Tesi_Michele_Fusilli.pdf

GALASSO, Giuseppe: En la periferia del Imperio. La monarquía hispánica y el reino de Nápoles, Barcelona, Península, 2000.

GARCILASO DE LA VEGA: Garcilaso de la Vega y sus comentaristas, ed. Antonio Gallego Morell, Madrid, Gredos, $1972^{2}$.

GARCILASO DE LA VEGA: Obra poética y textos en prosa, ed. Bienvenido Morros, Barcelona, Critica, 1995.

GARGANO, Antonio: "Parlar aspro: la Canción IV de Garcilaso y la tradición petrosa", en Pedro Ruiz Pérez (ed.): Canones críticos en la poesía de los Siglos de Oro, Vigo, Academia del Hispanismo, 2008, pp. 19-46.

GARGANO, Antonio: "De Sannazaro a Garcilaso: traducción y transcodificación (a propósito de la Égloga II)", en Pierre Civil, Antonio Gargano, Matteo Palumbo y y Encarnación Sánchez García (coords.): Fra Italia e Spagna. 
Napoli crocevia di cultura durante il vicereame, Napoli, Liguori, 2011, pp. 117-130.

GARGANO, Antonio: Con canto acordado. Estudios sobre la poesía entre Italia y España en los siglos XV-XVII, Sevilla, Universidad, 2012(a).

GARGANO, Antonio: “«Las estrañas virtudes y hazañas de los hombres». Épica y panegírico en la Égloga II de Garcilaso", Criticón, 115(2012) (b), pp. 11-43.

GUIJOSA, Manuel Isidro: "Íñigo de Ávalos y el texto del Bellum Gallicum de César en un escriptorio milanés", en A. Moreno Hernández (ed.): Julio César: textos, contextos y recepción. De la Roma clásica al mundo actual, Madrid, UNED, 2010, pp. 167-196.

HERNANDO SÁNCHEZ, Carlos José: "Poder y cultura en el renacimiento italiano. La biblioteca del virrey Pedro de Toledo", Cuadernos de Historia Moderna, 9(1980), pp. 13-33.

HERNANDO SÁNCHEZ: Carlos José: Castilla y Nápoles en el siglo XVI. El virrey Pedro de Toledo. Linaje, estado y cultura (1532-1553), Salamanca, Junta de Castilla y León, 1994.

HERNANDO SÁNCHEZ, Carlos José: "Parthénope ¿tan lejos de su tierra? Garcilaso de la Vega y la poesía de la corte en Nápoles”, en José María Díez Borque y Luis Ribor García (ed.): Garcilaso y su época: del amor y la guerra, Madrid, Sociedad Estatal de Conmemoraciones Culturales, 2003, pp. 71-141

HORACIO: Odas y Epodos, ed. bilingüe de Manuel Fernández Galiano y Vicente Cristóbal, Madrid, Cátedra, 1990.

LAPESA, Rafael: La trayectoria poética de Garcilaso, Madrid, Alianza, 1985.

LÁZARO CARRETER, Fernando: "La Ode ad florem Gnidi de Garcilaso de la Vega", en Víctor García de la Concha (ed.): Academia literaria renacentista. Garcilaso, Salamanca, Universidad, 1986, pp. 110-126.

LEFÈVRE, Matteo: "Garcilaso e Tansillo: una storia di amicizia e imitazione. La lirica tansiliana nella tradizione poetica spagnola", en Una poesia per l'Imperio. Lingua, editoria e tipologia del petrarchismo tra Spagna e Italia nell'epoca di Carlo V, Roma, Vecchiarelli editore, 2006(a), pp. 78-90.

LEFÈVRE, Matteo: "L'Accademia pontaniana, Bembo e Sannazaro. L'influenza dell'Arcadia sulla produzione bucolica di Garcilaso e sul petrarchismo spagnolo", en Una poesia per l'Imperio. Lingua, editoria e tipologia del petrarchismo tra Spagna e Italia nell'epoca di Carlo V, Roma, Vecchiarelli editore, 2006(b), pp. 60-78.

LEFÈVRE, Matteo: "Garcilaso de la Vega e la Napoli spagnola (1532-1536)", en Una poesia per l'Imperio. Lingua, editoria e tipologia del petrarchismo tra Spagna e Italia nell'epoca di Carlo V, Roma, Vechiarelli editore, 2006(c), pp. 55-59.

LOPEZ, Pasquale: Il movimento valdesiano a Napoli. Mario Galeota e le sue vicende al Sant'Ufizio, Napoli, Fausto Fiorentino, 1976. 
MELÉ, Eugenio: "Las poesías latinas de Garcilaso de la Vega y su permanencia en Italia", Bulletin Hispanique, 25,2(1923), pp. 108-148.

MENÉNDEZ PELAYO, Marcelino: Horacio en España, [Madrid], [Imprenta de A. Pérez Dubrull], $1885^{2}$.

MONFRIN, Jacques: "La bibliothèque des rois aragonais de Naples", Bibliothèque de l'École des chartes, 114, 1(1956), pp. 198-207.

MORROS, Bienvenido: "La muerte de Isabel Freyre y el amor napolitano de Garcilaso. Para una cronología de sus églogas y de otros poemas", Criticón, 105(2009), pp. 5-35.

PERCOPO, Erasmo: Artisti e scritori aragonesi, Napoles, Giannini, 1895.

PETRARCA, Francesco: Cancionero, ed. bilingüe de Jacobo Cortines, Barcelona, Cátedra, 1989.

PETROCCHI, Giorgio: Masuccio Guardati e la narrativa napoletana del quatrrrocento, Firenze, Le Monnier, 1953.

PIROVANO, Donato: Modi narrativi e stile del "Novellino" di Masuccio Salernitano, Firenze, La Nuova Italia, Editrice, 1996.

PONCE CÁRDENAS, Jesús: "Cauces de la imitación en el Renacimiento: Gutierre de Cetina y Nicolò Franco", en Mercedes Blanco y Roland Béhar (ed.): Les poètes de l'Empereur. La cour de Charles-Quint dans le renouveau littéraire $d u$ XVIe siècle (1516-1556), e-Spania, 13 junio (2012), sin paginación. Se lee en http://e-spania.revues.org/21485.

PROPERCIO: Poemas/Carmina, ed. bilingüe de Pedro Luis Cano Alonso, Barcelona, Bosch, 1994.

SALERNITANO, Masuccio, Il Novellino, ed. Alfredo Mauro [1940], pero cito por la reproducción de Salvatore S. Nigro, Roma/Bari, Laterza, 1975.

SANTAGATA, Marco: La lirica aragonese. Studi sulla poesia napoletana del secondo quattocento, Padova, Antenore, 1979.

SCOTTI, Mario: "Luigi Tansillo tra Rinascimento e Barocco", en Atti Convegno Internazionale sull'tema Premanirismo e Pregongorismo, Roma, Accademia Nazionale dei Lincei, 1973, pp. 125-150.

SLIWA, Krzysztof: Cartas, documentos y escrituras de Garcilaso de la Vega y sus familiares, Alcalá de Henares, Centro de Estudios cervantinos, 2006.

SPADA, Giovanni Battista: Giardino degli epiteti, Bologna, per l'erede de Vittorio Benaci, 1665.

TASSO, Bernardo: Rime, ed. Domenico Chiodo, Torino, Edizione RES, 1995.

TERRUSI, Leonardo "Masuccio Salernitano, Il Novellino", en P. Guaragnella y S. de Toma (ed.): Italian Studies, Lecce, Pensa Multimedia, 2011, I, pp. 149-159.

TOSCANO, Tobia R.: "Del reinado de Ferrante (1458-1494) hasta el final de la dinastía aragonesa", en Gennaro Toscano (ed.): La Biblioteca Reale di Napoli al tempo della dinastia aragonese, [Valencia], Consorci de Museus de la Comunitat Valenciana, [1998], pp. 147-167. 
TOSCANO, Tobia R.: "Fabio o Mario Galeota? Sull' identità di un rimatore napoletano del XVI secolo", Filologia e Critica, XXXV(2010), pp. 178-203.

TOSCANO, Tobia R.: "Tra corti e campo di battaglia: Alfonso d'Avalos, Luigi Tansillo e le affinità elettive tra petrarchisti napoletani e spagnoli", en Mercedes Blanco y Roland Béhar (ed.): Les poètes de l'Empereur. La cour de Charles-Quint dans le renouveau littéraire du XVIe siècle (1516-1556), eSpania, 13 junio (2012), sin paginación. Se lee en http://espania.revues.org/21485.

ZAMPESE, Cristine: Tevere e Arno. Studi sulla lirica del Cinquecento, Milano, Franco Angeli, 2012. 